\title{
Juvenile Chondrocytes May Facilitate Disc Repair
}

\author{
Anne J. Kim ${ }^{1}$, H. Davis Adkisson ${ }^{3}$, Michael Wendland ${ }^{2}$, Mitchell Seyedin ${ }^{3}$, Sigurd Berven ${ }^{1}$ and \\ Jeffrey C. Lotz ${ }^{*}, 1$ \\ ${ }^{I}$ Department of Orthopaedic Surgery, University of California, San Francisco. 533 Parnassus Ave, U-453. Box 0514, \\ San Francisco, CA 94143, USA \\ ${ }^{2}$ Department of Radiology, University of California, San Francisco. San Francisco, CA, USA \\ ${ }^{3}$ ISTO Technologies, Inc. St. Louis, MO 63132-3255, USA
}

\begin{abstract}
Cell transplantation for disc regeneration is a theoretically-attractive alternative to more invasive surgical procedures to treat discogenic pain. Several cell types have been tried in pre-clinical models for this indication, each with relative merits and limitations. Recently, human chondrocytes from prepubertal donors have been shown to be more synthetically active than their adult counterparts and can form scaffold-independent neocartilage in vitro. These features may also be beneficial in the intervertebral discs' challenging environment including high physical pressure and acidity, and low oxygen tension. To test this, we characterized the in vivo disc regeneration potential of juvenile chondrocytes in caudal rat discs. Human juvenile chondrocytes (JCs) were injected into fifteen rats. Fibrin glue without cells served as a control. Tails were imaged with MRI prior to sacrifice and tail discs were processed for histology. MRI data demonstrates that discs injected with fibrin glue alone degenerated over 12 weeks. By contrast, discs injected with JCs maintained their morphology and MRI signal, and displayed a significantly improved MRI index at 12 weeks post-injection. Transplantation of juvenile chondrocytes results in a significant positive outcome compared to that of fibrin glue alone and may provide an ideal allograft for future human studies.
\end{abstract}

Keywords: Intervertebral disc, tissue engineering, chondrocytes.

\section{INTRODUCTION}

Intervertebral disc degeneration is an underlying factor for a majority of spine-related disabilities leading to significant patient hardship and societal expense [1]. Patients who do not respond well to conservative therapy have few options other than fusion, which can result in considerable morbidity and variable outcomes [2]. While the focus of surgical and conservative treatments is to gain symptomatic relief, the underlying biological problem typically remains unidentified. The notion that back pain patients will benefit from less invasive procedures has led researchers to investigate several approaches of biologic disc repair.

The intervertebral disc (IVD) forms a portion of the joint between vertebral bodies and confers flexibility to the spine. The proteoglycan-rich extracellular matrix of the nucleus pulposus osmotically attracts water, creating a swelling pressure that is confined and therefore sustained by the fibrous lamellae of the annulus fibrosus. In health, spinal compressive loads are supported by the gel-like nucleus pulposus, while the annulus fibrosus serves as an intervertebral ligament.

As an avascular tissue, the disc has a limited ability to regenerate itself. The cellularity of the disc is also quite low,

*Address correspondence to this author at the Department of Orthopaedic Surgery, University of California, San Francisco. 533 Parnassus Ave, U453. Box 0514, San Francisco, CA 94143, USA; Tel: 415-476-7881; Fax: 415-476-1128; E-mail: lotzj@orthosurg.ucsf.edu with cells occupying less than $1 \%$ of the total disc volume [3]. The cells produce the extracellular matrix and are responsible for maintaining the balance between matrix breakdown and synthesis. Loss of cellularity and reduced extracellular matrix production is a central feature of degenerative disc disease, and consequently a tissue-engineering based method to heal the nucleus may be an effective treatment approach. The introduction of cells capable of producing and maintaining normal extracellular matrix in a high pressure, low oxygen tension, and low $\mathrm{pH}$ environment is critical for successful bioengineered disc regeneration.

Several different cell types have been investigated for intervertebral disc tissue engineering. Autologous disc cells (nucleus pulposus and annulus fibrosus) [4-6], chondrocytes $[7,8]$, or autologous chondrocyte-like cells obtained from disc [9] have been injected into various animal models and have demonstrated varying degrees of regenerative effects. However, difficulties with sourcing and expanding these cell populations have led to a search for alternative cell sources. Mesenchymal stem cells (MSCs) are an attractive cell type given they can be isolated from bone marrow or fat and have the potential to differentiate into a number of musculoskeletal tissues. However, once placed in the body MSC differentiation is influenced by a number of local environmental cues including growth factors, mechanical forces, cytokines, oxygen tension, and $\mathrm{pH}$ that may be beyond investigator control. Based on our investigation of MSCs in varying differentiated stages and their response to load [10], and studies comparing the response to load in undifferentiated cells and chondrocytes, we believe cells that are already 
differentiated toward the chondrocyte phenotype may be more stable relative to uncontrolled site factors and thereby respond more predictably than undifferentiated cells during the acute healing phase in vivo. To test this hypothesis, we injected proliferating chondrocytes harvested from juvenile human articular cartilage (juvenile chondrocytes) into the caudal disc space of rats using a fibrin glue carrier. Articular chondrocytes have phenotypic similarities to nucleus pulposus cells, and have been transplanted into humans with articular chondral defects [11]. Juvenile chondrocytes can be expanded in vitro and can produce neocartilage under conditions where adult articular chondrocytes cannot [12]. The rat-tail model has been used by many investigators to study the disc's degenerative response to injury and mechanical load [13-15], and will spontaneously degenerate after annular needle puncture [16]. Consequently, in the current study the ability of juvenile chondrocytes to prevent postpuncture degeneration was characterized by histology, MRI imaging, and PCR analysis at 2, 4 and 12 weeks after injection.

\section{METHODS}

\section{Materials}

Human juvenile chondrocytes (JCs) from a three-yearold male donor were provided by ISTO Technologies, Inc (St. Louis, MO). Briefly, these cells were isolated from enzymatically-digested knee cartilage within 24 hours of accidental death [12]. The cells were filtered through a 70 micron nylon strainer then expanded in T-150 culture flasks in Complete Serum-Free Medium to retain the chondrocyte phenotype. Finally, the chondrocytes were washed and resuspended to a density of $50 \times 10^{6}$ cells $/ \mathrm{mL}$ in CryoStor CS5 cryopreservation solution containing 5\% DMSO (BioLife Solutions, Bothell Wa), and stored in liquid nitrogen until use.

Thirty-six inbred Fisher rats (Simonsen Laboratories, Inc.) were used for the juvenile chondrocyte and fibrin injections, as JCs are reported to be immuno-privileged [17]. All experiments were approved by the UCSF Institutional Animal Care and Use Committee.

\section{Rat Coccygeal Disc Injections}

Rats were anesthetized with a mix of ketamine $(90 \mathrm{mg} / \mathrm{kg})$ and xylazine $(10 \mathrm{mg} / \mathrm{kg})$ and the injection site scrubbed with isopropyl alcohol and iodine. Buprenorphine $(0.03 \mathrm{mg} / \mathrm{kg})$ was administered perioperatively for pain. Human juvenile chondrocytes were suspended into a fibrin adhesive (Tisseel VH, Baxter Bioscience Corporation) at a concentration of $10^{7}$ cells $/ \mathrm{mL}$. An exact volume of $20 \mu \mathrm{L}$ of each component (fibrinogen and thrombin) of the fibrin adhesive was injected simultaneously using two separate 29 gauge needles inserted in direct opposition to one another as visualized under fluoroscopic guidance (Fig. 1). A total volume of $40 \mu \mathrm{L}$ was injected into each of four consecutive discs in an inbred Fisher rat. Some of the nucleus pulposus is displaced during this injection. Previous cell retention experiments show $55 \%$ of injectate is retained in the disc after injection, or 220,000 cells per disc. This cell amount is in excess of what is normally found in a rat disc nucleus pulposus [18]. Six rats were injected for each harvest time point ( 2 weeks, 4 weeks and 12 weeks). Additionally, six rats for each time point were injected with fibrin alone to serve as a control. Three rats injected with JCs for the 2week time point died shortly after injection, possibly due to pre-existing respiratory problems or poor reaction to anesthesia. These animals were not replaced.

\section{MRI Disc Assessment of Juvenile Chondrocyte-Injected Rats}

On the day of sacrifice, each rat was anesthetized with inhaled isofluorane (5\% isofluorane in $100 \% \mathrm{O}_{2}$ to induce, $1.5-2.5 \%$ thereafter to maintain), placed supine on a Plexiglas platform and warmed with a water-recirculated underpad. The tail was passed into a radiofrequency coil and the animal placed in a 1.5 Tesla magnet for MR imaging. The top three discs of each rat were imaged ( 8 consecutive $1 \mathrm{~mm}$ slices). Nucleus size and signal intensity were measured for each disc and a grade of 1 through 5 assigned based on these measurements. A grade of 1 corresponds to healthy discs (large nucleus and high signal intensity) and a grade of 5 corresponds to degenerated discs (small or absent nucleus, very low signal intensity).

\section{Disc Height Measurement}

X-rays (Faxitron X-ray Corp.) were taken for each animal prior to injection, immediately post-injection, and at sacrifice. Measurements were made on x-ray films using SPOT image capture software (Diagnostic Instruments, Inc.) The distance between vertebrae was measured at the center
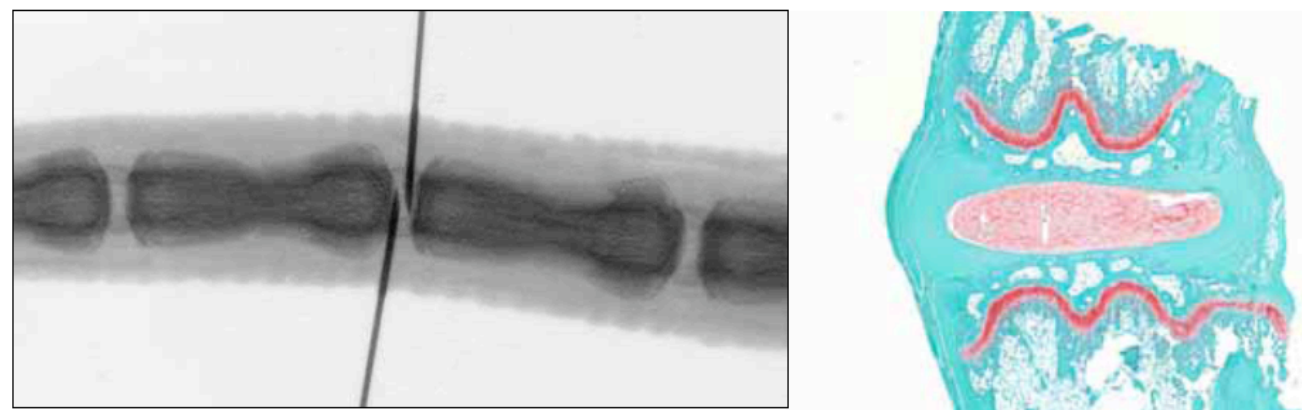

Fig. (1). X-ray image demonstrating fluoroscopic guidance used to monitor intradiscal 29 gauge needle placement during treatment (left). One needle was used for thrombin ( \pm chondrocytes) and the other for fibrinogen. The proximity of needle tips allows fibrin components to mix and polymerize upon contact. Safranin-O stained mid-sagittal section of normal tail disc demonstrates discrete nucleus and lamellar annular architecture (right). 
of each disc, and measurements were normalized to a fixedlength object imaged along side each tail. To control for animal size variability, the change in height (relative to the pre-operative value) was calculated.

\section{Tissue Structure, Cell Morphology, and Cell Retention}

At each time point, study discs were randomized into either a histology group $(\mathrm{n}=2)$ or an RNA analysis group $(n=2)$. Discs for histology were fixed in $10 \%$ neutral buffered formalin, decalcified with formic acid and embedded with paraffin. Seven $\mu$ m sagittal sections were cut from the entire disc and five representative slides spanning each disc were stained with Safranin-O/fast green to observe cell phenotype and matrix architecture. RNA analysis was used to confirm the presence of the injected cells by identifying human-specific $\beta_{2}$ microglubulin $\left(\beta_{2} \mathrm{~m}\right)$. Discs were harvested at sacrifice and flash frozen. RNA extraction of combined tissues ( 2 discs per rat) was performed using the TissueRuptor (handheld rotor-stator homogenizor) and RNeasy Fibrous Tissue Mini Kit purchased from Qiagen. Total amounts of RNA extracted ranged from $0.98 \mu \mathrm{g}$ to $3.32 \mu \mathrm{g}$. All reverse transcription and PCR reagents were purchased from Invitrogen. Primers were manufactured by Integrated DNA Technologies. DNase digestion was performed prior to reverse transcription to eliminate contaminating DNA. Two microliters of cDNA were used for a $50 \mu \mathrm{L}$ PCR reaction with a primer concentration of $0.2 \mu \mathrm{M}$. For amplification of $\beta_{2} \mathrm{~m}$, PCR was performed as described previously [19] with an initial denaturation for 2 $\min$ at $94^{\circ} \mathrm{C}$, followed by 35 cycles of $94^{\circ} \mathrm{C}$ for $30 \mathrm{~s}, 55^{\circ} \mathrm{C}$ for $30 \mathrm{~s}$ and $72^{\circ} \mathrm{C}$ for $1 \mathrm{~min}$, with a final extension for $10 \mathrm{~min}$ at $72^{\circ} \mathrm{C}$. The primers $\left(5^{\prime}\right.$-ATA TCC ATA TCC ATA TGT CTC GCT CCG TGG CCT TAG-3' and 5'-AAC TAG GGA TCC TTA CAT GTC TCG ATC CCA C-3') amplify the entire coding sequence of human $\beta_{2}$ m cDNA. PCR products were separated and visualized on a $1 \%$ agarose gel stained with ethidium bromide. The $\beta_{2} \mathrm{~m}$ product size of $357 \mathrm{bp}$ was measured against a 100bp DNA ladder (New England BioLabs Inc.). Samples of control rat discs mixed with a range of 10,000 to 2 million human MSCs were homogenized and used for PCR to determine if low numbers of cells could be detected. Cell amounts of 50,000 cells or greater were sufficient to yield enough cDNA that was detectable by PCR and gel electrophoresis.

\section{Data Analysis}

One-way analysis of variance was used to test for statistically significant differences in disc height between groups. When appropriate, a Tukey post-hoc test was used for pairwise comparisons. The Mann-Whitney rank-sum non-parametric test was used to analyze the MRI data and to test whether cell treatment resulted in a statisticallysignificant improvement in disc grade.

\section{RESULTS}

\section{Change in Disc Height}

At two weeks after injection, disc heights measured for cell-injected discs were significantly decreased compared to that of control discs receiving fibrin alone (Fig. 2, $\mathrm{p}<0.01$ ). In control discs, disc heights did not change significantly at increasing time points. However, disc height was significantly restored at four and twelve weeks compared to two weeks in cell-injected discs $(\mathrm{p}<0.001)$.

\section{MRI Signal}

At four and twelve weeks, control rats presented with more degenerated discs (grade four and five) than JCinjected rats, and the $\mathrm{JC}$-injected rats had more healthy discs

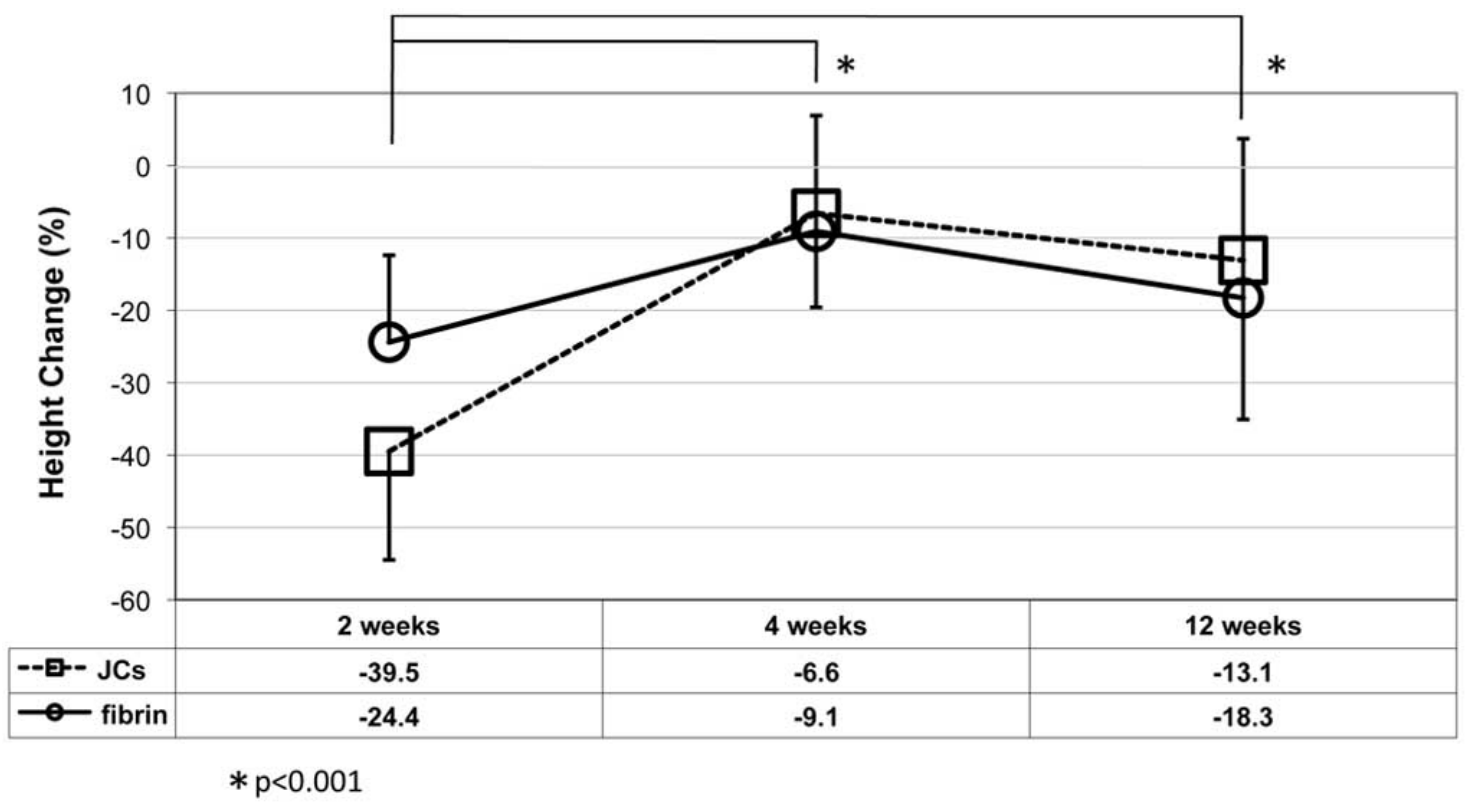

Fig. (2). Disc height change relative to its pre-operative value as measured using $x$-ray images (means \pm standard deviation). Height was significantly restored at 4 and 12 weeks in JC-injected discs when compared to two weeks $(\mathrm{p}<0.001)$. Discs treated with fibrin alone showed an initial $24.4 \%$ reduction in disc height, but this transient loss of disc height appeared to be restored, in large part, by and 12 weeks to levels that were nearly equivalent to JC-injected discs. 


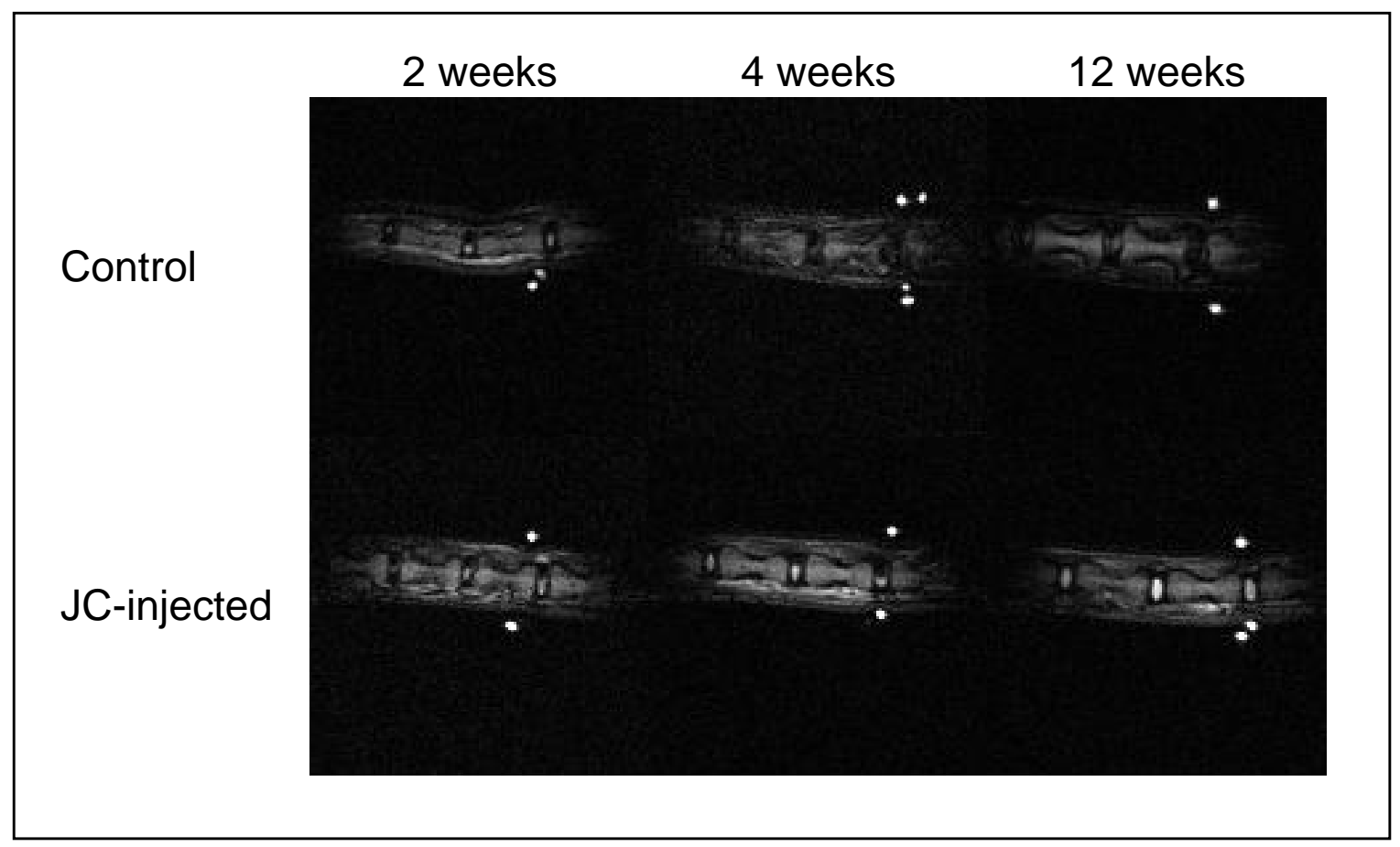

Fig. (3). Sequential T2-weighted MRI of rat caudal discs. Three adjacent discs per tail were injected with the same treatment. For reference purposes, the exterior of the tail at the most caudal treated disc was encircled with a MR contrast filled polymer tube. The increased nuclear signal intensity (water content) over time demonstrated a positive healing effect with JC injection. Treatment with fibrin alone resulted in a marked reduction in signal intensity between weeks 2 and 12, discs receiving JCs showed marked restoration of signal intensity.

(grade 1) than control rats (Table 1). At four weeks, MRI grade was significantly improved in JC-injected discs $(\mathrm{p}<0.02)$. While more than half of the control discs appeared to degenerate over twelve weeks, all but two of the 18 imaged discs injected with JCs maintained morphology, as measured by MRI signal intensity (Fig. 3).

Table 1. Number of Discs Per MRI Degeneration Grade Per Treatment Group

\begin{tabular}{|c|c|c|c|c|c|c|}
\hline \multirow{2}{*}{ Grade } & \multicolumn{2}{|c|}{ weeks } & \multicolumn{2}{c|}{ 4 weeks } & \multicolumn{2}{c|}{12 weeks } \\
\cline { 2 - 7 } & Cells & Control & Cells & Control & Cells & Control \\
\hline \hline 1 & 0 & 0 & 7 & 1 & 3 & 0 \\
\hline 2 & 3 & 7 & 3 & 6 & 6 & 8 \\
\hline 3 & 3 & 7 & 7 & 5 & 6 & 3 \\
\hline 4 & 1 & 3 & 1 & 1 & 1 & 0 \\
\hline 5 & 2 & 1 & 0 & 5 & 2 & 7 \\
\hline
\end{tabular}

For each disc, the MRI grade was measured from the MRI images and assigned a value from 1 to 5 , where 1 is normal and 5 is very degenerate.

\section{Histological Analysis}

At two weeks, discs from both groups show the effects of the injection with disrupted annular architecture and reduced nucleus size (Fig. 4). In more than half of treated discs, remnants of native nucleus are visible along the wound tract and pushed outside the annulus. While inflammatory cells were not present within the cell-injected and control discs, some inflammatory cells were observed to be present at the point of needle entry outside the annulus (Fig. 5).

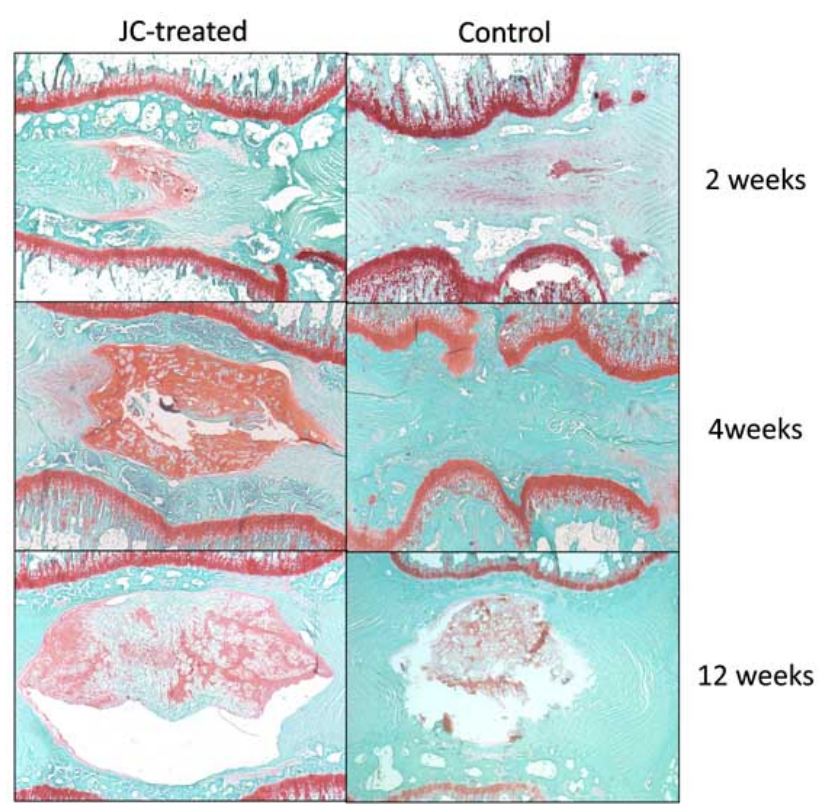

Fig. (4). Safranin-O stained mid-sagittal histologic sections of a representative discs $(4 \mathrm{x})$. Control discs demonstrate a marked reduction in Safranin-O content in the nucleus pulposus and associated loss of disc height (right column). Maintained SafraninO nuclear staining was observed in JC-treated discs (left column). No evidence of inflammation was observed for any treatment group at 12 weeks post-intervention.

At four weeks, differences in the morphological appearance of control and cell-injected discs are more apparent. 

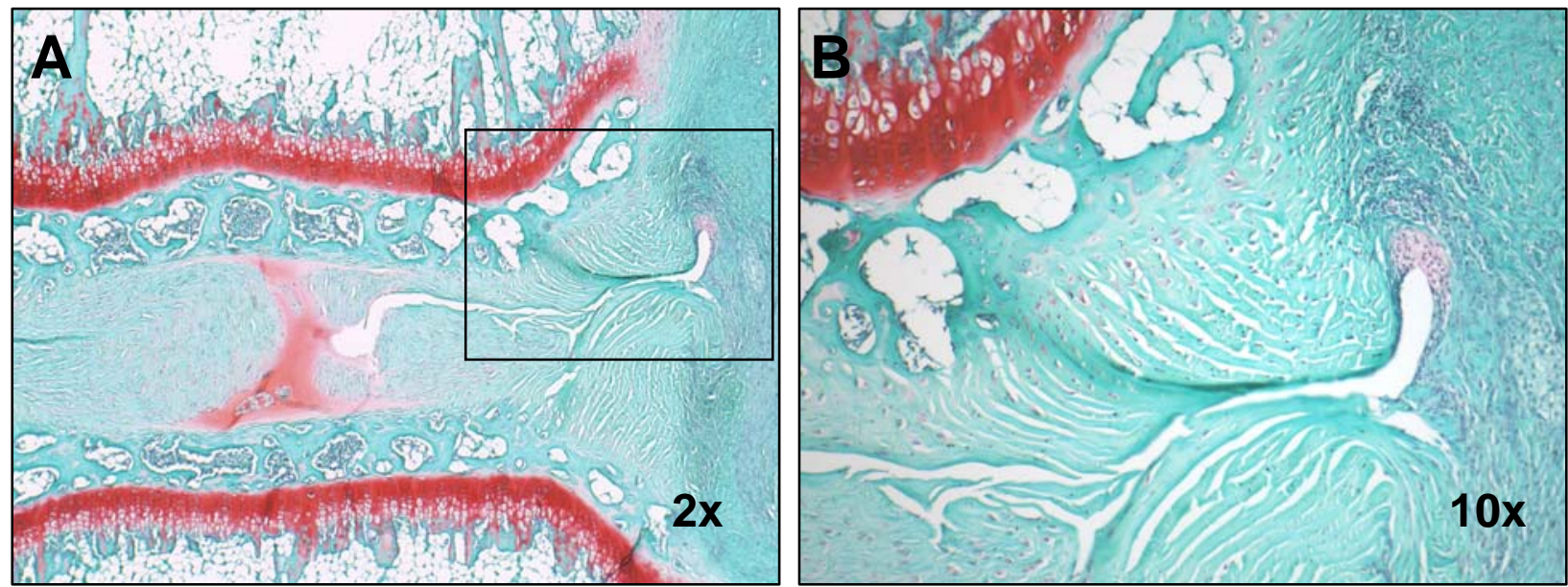

Fig. (5). Safranin-O stained mid-sagittal histologic sections of a representative JC-treated disc at 2 weeks after injection. At low power (A; $2 \mathrm{x})$ granulation tissue is apparent outside the annular wound tract. At high power $(\mathbf{B} ; 10 \mathrm{x})$ there is absence of any inflammatory cells at the injection site; newly synthesized hyaline matrix is typically observed in the wound tract.

Eight out of the 12 control discs $(66.7 \%)$ show remnants of nucleus outside the annulus, and six out of the 12 discs $(50 \%)$ have disrupted annular architecture along the wound tract. For JC-injected discs, only two out of 12 discs (16.7\%) have disrupted annular architecture along the wound tract; the remaining discs show no evidence of the wound tract. JC-injected discs show evidence of regaining normal round nuclear structure (Fig. 4) while nine out of the 12 control discs $(75 \%)$ have irregular nucleus shape and disrupted inner annular architecture as a result of loss of nuclear material.

At twelve weeks, six out of the 12 control discs $(50 \%)$ were severely degenerated, with complete absence of proteoglycan staining in the nucleus and an irregular annulus structure. The remaining six control discs presented with reduced nucleus size and moderate proteoglycan staining. In contrast, four of the $12 \mathrm{JC}$-injected discs (33.3\%) appeared to regain normal nucleus size, and six of 12 discs $(50 \%)$ presented with normal nucleus size but slightly irregular shape. One JC-injected disc was severely degenerated but this appeared to be due to endplate damage during the initial injection.

Discs injected with JCs demonstrated chondrocyte-like cells proliferating at the inner annular/nuclear border (Fig. 6). These cells were not visible in the six control discs that were not severely degenerated.

\section{Cell Retention}

Injected JCs were confirmed present at all time points by PCR. Human $\beta_{2} \mathrm{~m}$ products were not detected in any of the control rats and were detected in all three JC-injected rats at two weeks, three of the six treated rats at four weeks, and five of the six treated rats at twelve weeks (Fig. 7). The absence of human $\beta_{2} \mathrm{~m}$ products in some JC-injected rats could be due to low cell numbers that led to difficulty in detection by PCR.
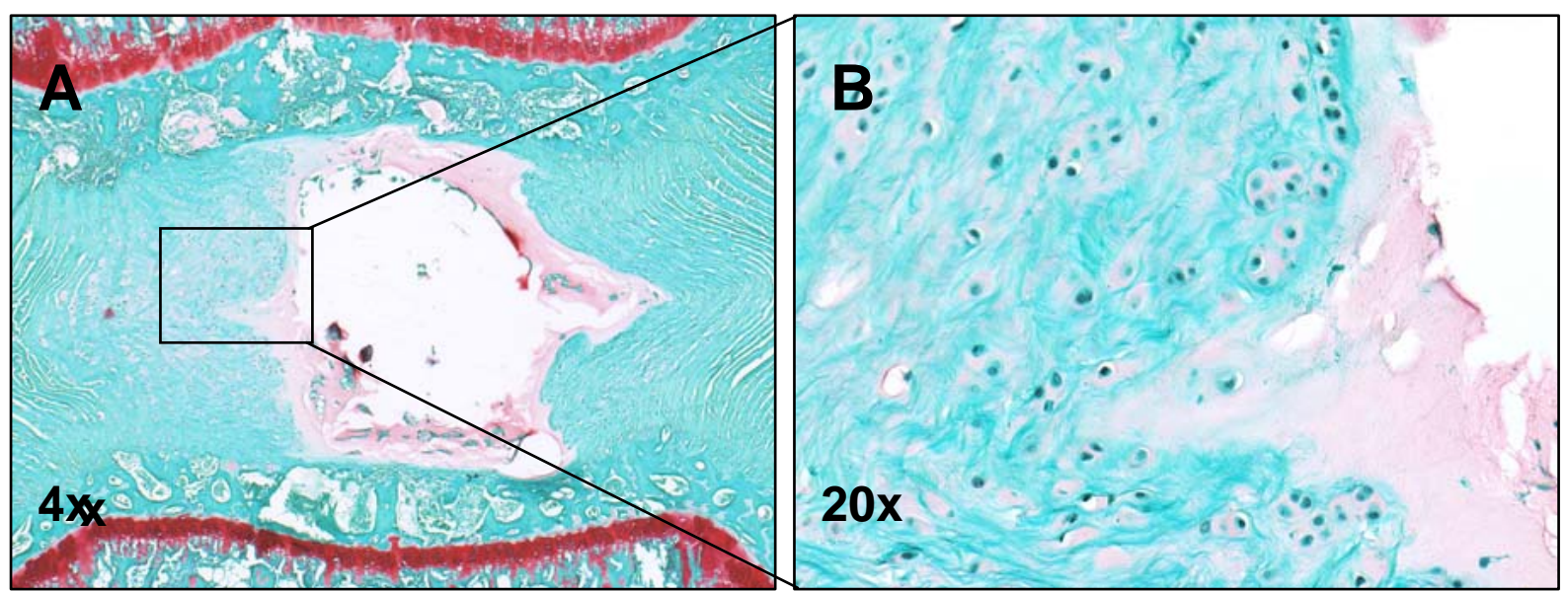

Fig. (6). Safranin-O stained mid-sagittal histologic section of representative JC-treated disc harvested 12 weeks post-injection (A). Engrafted chondrocytes are observed at the inner annulus border for JC-injected discs (B). 


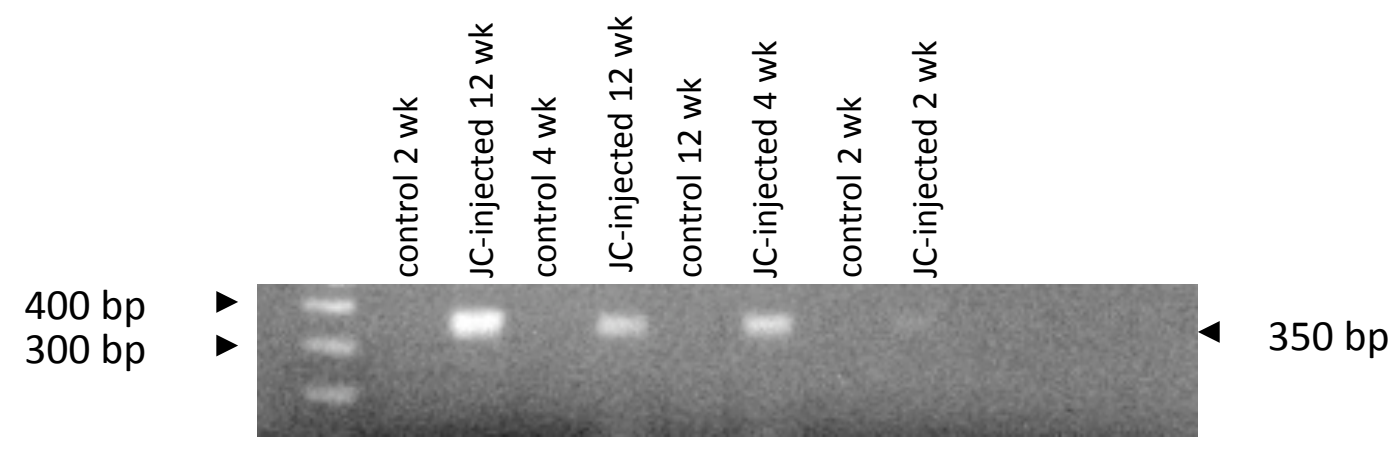

Fig. (7). RT-PCR results confirming presence of human specific $\beta 2$-microglobulin transcript (amplified fragment $=357 \mathrm{bp}$ ) in JC-injected discs at 2, 4 and 12 weeks post-injection.

\section{DISCUSSION}

We hypothesized that synthetically-active chondrocytes from prepubertal human donors would respond favorably in the disc environment. We observed that in vivo transplantation of juvenile chondrocytes in a fibrin carrier results in a positive outcome relative to fibrin alone. Since these cells are non-immunogenic, may be expanded in vitro [12], and respond well to in vivo disc conditions [20], they may provide an ideal source of cells for future human disc repair studies.

Although the disc height data suggests there is no difference between control and cell-injected discs at 4 and 12 weeks, histology reveals that discs injected with cells have normal-sized nucleus but a slightly flattened appearance, explaining the slight reduction in disc height. Nucleus size and signal intensity data from MRI for corresponding discs also confirm this.

Histologic data illustrates further differences between cell-injected and control discs. Discs injected with JCs appear to have faster wound tract healing than control discs. Fewer or no cell-injected discs display persistence of the wound tract, nuclear remnants, and disrupted annular architecture at time points where these features were still present in fibrin-only discs. At four weeks post-injection, most control discs still have nuclear material in the outer annulus or outside the annulus, while most of the cellinjected discs lack this feature and are regaining normal nucleus size (Fig. 5). This is supported by the significant increase in MRI grade at four weeks for JC-injected discs. A possible explanation for this observation may be that JCs express bone morphogenetic proteins (BMPs) that have been implicated in accelerating cartilage repair [21], inducing chondrogenesis $[22,23]$, and stimulating proteoglycan and collagen synthesis by nucleus pulposus cells in vitro [24].

Limitations of this study relate to challenges of the animal model used. First, it has been well established in the literature that the intervertebral disc presents a harsh environment (high pressure, low oxygen and low $\mathrm{pH}$ ) to newly implanted cells, and this environment can become more challenging in a degenerated disc [3, 25-27]. We used a healthy intervertebral disc model and the response of injected cells may differ in a degenerated disc environment. Secondly, although the amount of fibrin (with or without cells) injected was constant for all discs, the amount of nucleus displaced by the injection is variable. The effects of this variability are most evident in the 12 -week histological data. Although half of the fibrin-injected control discs show complete degeneration, many also show signs of regeneration as determined by increased proteoglycan staining, suggesting that a smaller amount of nucleus was displaced during the treatment injection and can thereby participate in new matrix synthesis. It is important to note the differences between these "spontaneously regenerated" discs and discs injected with JCs. The presence of proliferating chondrocytes at the border of the inner annulus and nucleus in the JCinjected discs suggest a more active remodeling process compared to discs without cell treatment. This phenomenon has been demonstrated in vitro by others. Co-culture of nucleus pulposus cells with either mesenchymal stem cells or BMP-transduced articular chondrocytes resulted in increased proliferation and proteoglycan synthesis by the NP cells [24, 28]. Additionally, Le Visage and coworkers report that MSCs cultured in contact with annulus fibrosus cells resulted in overall enhanced proteoglycan synthesis [29].

Richardson and coworkers report that the increase in proteoglycan synthesis during MSC/NP co-culture is due to NP-mediated MSC differentiation [30]. This raises questions as to which cell types could be best suited for intervertebral disc repair. Several researchers have demonstrated the feasibility of injecting mesenchymal stem cells, intervertebral disc cells, or chondrocytes into the disc for repair [4-8, 31, 32 ], yet none have addressed which type of cell would be most beneficial. Our findings reported here support the notion that differentiated cells are receptive to the disc milieu. This observation is consistent with prior studies demonstrating significant differences between undifferentiated cells and chondrocytes in terms of their response to physical stimuli [33]. Response to compression is more pronounced in chondrocytes, where the level of matrix development is more advanced [34]. Although MSCs may ultimately differentiate in the disc and ultimately secrete proteoglycan, the quality of matrix secreted may not be sufficient for disc regeneration. For example, MSCs in three-dimensional culture have reduced potential to form functional matrix compared to similarly cultured chondrocytes, where chondrocytes formed matrix that had two- to three-fold higher mechanical properties than that from MSCs [35]. Even when given additional culture time to allow for possible differentiation, MSC constructs never caught up to chondrocytes in terms of 
mechanical properties and proteoglycan synthesis. MSCs grown in 3-D culture also required chondrogenic media to remain viable.

Other conditions of the degenerated disc such as low oxygen tension and inflammatory cytokine production may influence cell behavior and differentiation. Inflammatory cytokines such as IL- $1 \beta$ and TNF- $\alpha$ have been shown to interfere with chondrogenesis, inhibit collagen and proteoglycan synthesis, reduce TGF- $\beta$-induced SMAD binding, or decrease Sox9 expression in chondrocytes [36-38]. Low oxygen tension can either inhibit or enhance in vitro chondrogenesis, depending on the cell type used [39, 40]. A combination of low oxygen tension and pressure was reported to enhance aggrecan and collagen production by cultured chondrocytes, but did not have the same effect on bone marrow-derived stem cells [40]. These factors affecting cell fate post-implantation should be taken into account in future in vitro experiments aiming to identify an optimal cell source for therapeutic application in the disc, and the effects of pressure, oxygen tension, and inflammatory cytokines should be explored in concert.

Future studies should also address cell fate in pre-clinical models that are more representative of the future clinical indication. Yet, the markers of painful discs are ill defined. Recently, we have reported chemical spectra from disc samples removed at surgery that sheds light on pain-specific disc features [41]. Tissues from patients with painful discs (treated with disc arthroplasty or fusion) differ significantly from patients without disc pain (adults treated for scoliosis) - pain patients have a clear decrease in the ratio of proteoglycan to lactate. This observation fits well with the theoretical view that because the disc is avascular, its cells may easily enter anaerobic metabolism when there is an imbalance between metabolic demands and nutritional support. Also, elevated lactate is a known marker of active wound healing and a stimulus of acid-sensing neurons [42]. These observations support the view that painful discs are characterized by damage accumulation (due to repetitive activities of daily living) superimposed on an inadequate wound healing response [43]. Ultimately leading to persistent inflammation [14]. If this proves true, a central therapeutic goal of disc tissue engineering should be to enhance wound healing, which includes increasing matrix synthesis while decreasing inflammation.

\section{REFERENCES}

[1] Ehrlich GE. Low back pain. Bull World Health Organ 2003; 81(9): 671-6.

[2] Turner JA, Ersek M, Herron L, Haselkorn J, Kent D, Ciol MA, et al. Patient outcomes after lumbar spinal fusions. J Am Med Assoc 1992; 268(7): 907-11.

[3] Bibby SR, Jones DA, Lee RB, Yu J, Urban JPG. The pathophysiology of the intervertebral disc. Joint Bone Spine 2001; 68(6): 537-42.

[4] Okuma M, Mochida J, Nishimura K, Sakabe K, Seiki K. Reinsertion of stimulated nucleus pulposus cells retards intervertebral disc degeneration: an in vitro and in vivo experimental study. J Orthop Res 2000; 18(6): 988-97.

[5] Watanabe K, Mochida J, Nomura T, Okuma M, Sakabe K, Seiki K. Effect of reinsertion of activated nucleus pulposus on disc degeneration: an experimental study on various types of collagen in degenerative discs. Connect Tissue Res 2003; 44(2): 104-8.
[6] Gruber HE, Johnson TL, Leslie K, et al. Autologous intervertebral disc cell implantation: a model using Psammomys obesus, the sand rat. Spine 2002; 27(15): 1626-33.

[7] Gorensek M, Jaksimovic C, Kregar-Velikonja N, et al. Nucleus pulposus repair with cultured autologous elastic cartilage derived chondrocytes. Cell Mol Biol Lett 2004; 9(2): 363-73.

[8] Zhang Y, Phillips FM, Thonar EJ, Oegema T, An HS, Roman-Blas $\mathrm{JA}$, et al. Cell therapy using articular chondrocytes overexpressing BMP-7 or BMP-10 in a rabbit disc organ culture model. Spine (Phila Pa 1976) 2008; 33(8): 831-8.

[9] Ganey T, Libera J, Moos V, et al. Disc chondrocyte transplantation in a canine model: a treatment for degenerated or damaged intervertebral disc. Spine 2003; 28(23): 2609-20.

[10] Haudenschild AK, Hsieh AH, Kapila S, Lotz JC. Pressure and distortion regulate human mesenchymal stem cell gene expression. Ann Biomed Eng 2009; 37(3): 492-502.

[11] Marcacci M, Kon E, Zaffagnini S, et al. Arthroscopic second generation autologous chondrocyte implantation. Knee Surg Sports Traumatol Arthrosc 2007; 15(5): 610-9.

[12] Adkisson HD, Gillis MP, Davis EC, Maloney W, Hruska KA. In vitro generation of scaffold independent neocartilage. Clin Orthop Relat Res 2001; 391(Suppl): S280-94.

[13] Iatridis JC, Mente PL, Stokes IA, Aronsson DD, Alini M. Compression-induced changes in intervertebral disc properties in a rat tail model. Spine 1999; 24(10): 996-1002.

[14] Ulrich JA, Liebenberg EC, Thuillier DU, Lotz JC. ISSLS prize winner: repeated disc injury causes persistent inflammation. Spine 2007; 32(25): 2812-9.

[15] Rousseau MA, Ulrich JA, Bass EC, Rodriguez AG, Liu JJ, Lotz JC. Stab incision for inducing intervertebral disc degeneration in the rat. Spine 2007; 32(1): 17-24.

[16] Zhang KB, Zheng ZM, Liu H, Liu XG. The effects of punctured nucleus pulposus on lumbar radicular pain in rats: a behavioral and immunohistochemical study. J Neurosurg 2009; 11(4): 492-500.

[17] Adkisson HD, Milliman C, Zhang X, Mauch K, Maziarz RT, Streeter PR. Immune evasion by neocartilage-derived chondrocytes: Implications for biologic repair of joint articular cartilage. Stem Cell Res 2010; 4: 57-68.

[18] Latorre A, Albareda J, Castiella T, Lasierra JM, Seral F Experimental model of multidirectional disc hernia in rats. Int Orthop1998; 22(1): 44-8.

[19] $\mathrm{He} \mathrm{XH}, \mathrm{Xu}$ LH, Liu Y. Procedure for preparing peptide-major histocompatibility complex tetramers for direct quantification of antigen-specific cytotoxic T lymphocytes. World J Gastroenterol 2005; 11(27): 4180-7.

[20] Kim AJ, Adkisson M, Wendland M, Lotz JC. Juvenile chondrocytes may facilitate disc repair. 53rd Annual Meeting of the Orthopaedic Research Society. San Diego, CA 2007.

[21] Hidaka C, Goodrich LR, Chen CT, Warren RF, Crystal RG, Nixon AJ. Acceleration of cartilage repair by genetically modified chondrocytes over expressing bone morphogenetic protein-7. J Orthop Res 2003; 21(4): 573-83.

[22] Jin EJ, Lee SY, Choi YA, Jung JC, Bang OS, Kang SS. BMP-2enhanced chondrogenesis involves p38 MAPK-mediated downregulation of Wnt-7a pathway. Mol Cells 2006; 22(3): 353-9.

[23] Estes BT, Wu AW, Guilak F. Potent induction of chondrocytic differentiation of human adipose-derived adult stem cells by bone morphogenetic protein 6. Arthritis Rheum 2006; 54(4): 1222-32.

[24] Zhang YG, Guo X, Xu P, Kang LL, Li J. Bone mesenchymal stem cells transplanted into rabbit intervertebral discs can increase proteoglycans. Clin Orthop Relat Res 2005; 430: 219-26.

[25] Antoniou J, Steffen T, Nelson F, et al. The human lumbar intervertebral disc: evidence for changes in the biosynthesis and denaturation of the extracellular matrix with growth, maturation, ageing, and degeneration. J Clin Invest 1996; 98(4): 996-1003.

[26] Buckwalter JA. Aging and degeneration of the human intervertebral disc. Spine 1995; 20(11): 1307-14.

[27] Ohshima H, Urban JPG. The effect of lactate and $\mathrm{pH}$ on proteoglycan and protein synthesis rates in the intervertebral disc. Spine 1992; 17(9): 1079-82.

[28] Yamamoto Y, Mochida J, Sakai D, et al. Upregulation of the viability of nucleus pulposus cells by bone marrow-derived stromal cells: significance of direct cell-to-cell contact in coculture system. Spine 2004; 29(14): 1508-14.

[29] Le Visage C, Kim SW, Tateno K, Sieber AN, Kostuik JP, Leong $\mathrm{KW}$. Interaction of human mesenchymal stem cells with disc cells: 
changes in extracellular matrix biosynthesis. Spine (Phila Pa 1976) 2006; 31(18): 2036-42.

[30] Richardson SM, Walker RV, Parker S, et al. Intervertebral disc cell-mediated mesenchymal stem cell differentiation. Stem Cells (Dayton, Ohio) 2006; 24(3): 707-16.

[31] Sakai D, Mochida J, Iwashina T, et al. Regenerative effects of transplanting mesenchymal stem cells embedded in atelocollagen to the degenerated intervertebral disc. Biomaterials 2006; 27(3): $335-45$.

[32] Sakai D, Mochida J, Iwashina $\mathrm{T}$, et al. Differentiation of mesenchymal stem cells transplanted to a rabbit degenerative disc model: potential and limitations for stem cell therapy in disc regeneration. Spine (Phila Pa 1976) 2005; 30(21): 2379-87.

[33] Takahashi I, Nuckolls GH, Takahashi K, et al. Compressive force promotes sox9, type II collagen and aggrecan and inhibits IL-1beta expression resulting in chondrogenesis in mouse embryonic limb bud mesenchymal cells. J Cell Sci 1998; 111 (Pt 14): 2067-76.

[34] Buschmann MD, Gluzband YA, Grodzinsky AJ, Hunziker EB. Mechanical compression modulates matrix biosynthesis in chondrocyte/agarose culture. J Cell Sci 1995; 108(Pt 4) (4): 1497508.

[35] Mauck RL, Yuan X, Tuan RS. Chondrogenic differentiation and functional maturation of bovine mesenchymal stem cells in longterm agarose culture. Osteoarthritis Cartilage 2006; 14(2): 179-89.
[36] Mohamed-Ali H. Influence of interleukin-1 beta, tumour necrosis factor alpha and prostaglandin E2 on chondrogenesis and cartilage matrix breakdown in vitro. Rheumatol Int 1995; 14(5): 191-9.

[37] Murakami S, Lefebvre V, de Crombrugghe B. Potent inhibition of the master chondrogenic factor Sox9 gene by interleukin-1 and tumor necrosis factor-alpha. J Biol Chem 2000; 275(5): 3687-92.

[38] Roman-Blas JA, Stokes DG, Jimenez SA. Modulation of TGF-beta signaling by proinflammatory cytokines in articular chondrocytes. Osteoarthritis Cartilage 2007; 15(12): 1367-77.

[39] Malladi P, Xu Y, Chiou M, Giaccia AJ, Longaker MT. Effect of reduced oxygen tension on chondrogenesis and osteogenesis in adipose-derived mesenchymal cells. Am J Physiol Cell Physiol 2006; 290(4): C1139-46.

[40] Scherer K, Schunke M, Sellckau R, Hassenpflug J, Kurz B. The influence of oxygen and hydrostatic pressure on articular chondrocytes and adherent bone marrow cells in vitro. Biorheology 2004; 41(3-4): 323-33.

[41] Keshari KR, Lotz JC, Link TM, Hu SS, Majumdar S, Kurhanewicz J. Lactic acid and proteoglycans as metabolic markers for discogenic back pain. Spine 2008; 33(3): 312-7.

[42] Hunt TK, Aslam RS, Beckert S, et al. Aerobically derived lactate stimulates revascularization and tissue repair via redox mechanisms. Antioxid Redox Signal 2007; 9(8): 1115-24. .

[43] Lotz JC, Ulrich JA. Innervation, inflammation, and hypermobility may characterize pathologic disc degeneration: review of animal model data. J Bone Joint Surg 2006; 88(Suppl 2): 76-82.

(C) Kim et al.; Licensee Bentham Open.

This is an open access article licensed under the terms of the Creative Commons Attribution Non-Commercial License (http://creativecommons.org/ licenses/by-nc/3.0/), which permits unrestricted, non-commercial use, distribution and reproduction in any medium, provided the work is properly cited. 